\title{
Contingent Consideration of Lightweight Algorithms for IoT
}

\author{
S Pradeep ${ }^{\# 1}$, Saideepthi Pabba ${ }^{* 2}$, K Sudhakar ${ }^{\# 3}$ \\ ${ }^{1}$ Associate Professor, Dept of Computer Science \& Engg, \\ Bhoj Reddy Engineering College for Women, Hyderabad, Telangana, India. \\ ${ }^{2}$ Assistant Professor, Dept of Computer Science \& Engg, \\ Saideepthi Pabba , Former Asst. Professor K J Somaiya College of Engineering, India. \\ ${ }^{3}$ Associate Professor, Dept of Electronic Communication Engg, \\ Jayaprakash Narayan College of Engineering, Mahabubnagar, Hyderabad, Telangana, India. \\ ${ }^{1}$ pradeep.sunkari87@gmail.com
}

\begin{abstract}
IOT things, most of the course world applications are choosing IOT approach as it makes them commutable, efficient and fast. However, IoT devices are interconnected and persist across wider geographical locations .hence it coexists and leads to a greater security risk. As a result of this, it is at most important to analyze the existing cryptographic ciphers on such devices and decide, if they are feasible for expression implementation process and memory utilization efficiency . Security \& privacy are the crucial element which needs to be addressed to hold upto the faith in consumer of Internet of things . Existing solutions for protecting to every sheet will be a vulnerable to problem. Therefore, lightweight cryptographic solutions are defined as an alternative solution to strengthen security. This paper provides an analysis on existing lightweight cryptographic algorithm which are used for providing security in IoT
\end{abstract}

Keyword - Internet of things, lightweight cryptography, Information security, throughput, efficiency

\section{INTRODUCTION}

Today's world depends for the data reserves to the point of Communication of internet, in the form of captured images or text .Here human being is involved for collection of the information .But the main disadvantage with the human involvement is that, less accuracy and limited time, which might lead to inconsistent and inappropriate data. Henceforth a system chooses accordingly grasping the information and deportation it to the data externally to any individual to appliance communication in desired. Currently the impact of internet in science, communication ,business, education ,government and humanity is huge and notable .Internet is found to be the most important and powerful creation in human history .Now with the concept of internet of things being widely used .internet has become more favourable and needed to have a smart life in every aspect

\section{IOT SECURITY ARCHITECTURE}

The Common planning line of credible preservation arrangement located on Internet of things is described by Xiong Li Zhou Xuan .The IoT architecture is divided into 4 layers : 1.Preception Segment 2.Network Segment 3.Conceptual division segment and 4.Abstract Segment

\begin{tabular}{|c|}
\hline $\begin{array}{c}\text { Abstract Segment } \\
\text { (Applications /Devices) }\end{array}$ \\
\hline $\begin{array}{c}\text { Conceptual division Segment } \\
\text { (End - End Communication. Securitv) }\end{array}$ \\
\hline $\begin{array}{c}\text { System of Connection Segment } \\
\text { (Wired /Wireless network, Other Network Components) }\end{array}$ \\
\hline Perception Segment \\
(Sensors, RFID tags, Smart Cards ) \\
\hline
\end{tabular}

Fig 1: Architecture of IOT 


\section{Perception Layer:}

It is responsible for data collection which is the main working of IoT. In this process we find the RFID tag, smart card, reader and sensor networks been taken to collect the data from the end devices. Perception layer relates to sensing which reflects the object to identify information from anytime and anywhere.

\section{Network Layer:}

The Date acquisition by the sensors is forwarded towards the computer data over network segment with the end devices, communicating without material contact network and related elements

\section{Transport Layer:}

It is answering for two process application specific communication of Internet of Things proceeds with mechanism of Routing information Protocol. Safety tool using Datagram Transport Layer Security for making towards the layer.

\section{Application Layer:}

It analyses for gathering data and makes the control decisions. This layer as the identification and control between objects and devices connections.

TABLE 1: Process Layer

\begin{tabular}{|c|c|c|c|}
\hline Layer & Protocol & Security Protocol & Attacks \\
\hline Perception & IEEE 802.15.4 MAC & IEEE 802.15.4 Security & Dos-Attack ,Authentication \& Integrity \\
\hline Network & IPV6,RPL & IPSec & Dos Attack \\
\hline Transport & UDP & DTLS & Attack-on RC4,Dos Attack \\
\hline Application & COAP & Designed by User & Depends-on protocol used \\
\hline
\end{tabular}

\section{FRAMES OF SECURITY}

A. IoT Security Goals:

1. Confidentiality: Authorized Objects

2. Integrity: Data Completeness and Accuracy.

3. Non-Repudiation: Communicating device with incident and Non-incident of an event

4. Availability: IoT Service are easily accessible by authorized objects.,

5. Privacy: Privacy and Policies allows users to control sensitive data

6. Accountability: IoT user with responsible actions.

B. Security Attacks:

1. Man-in-Middle: It is Intermediate user to start communication as valid party.

2. Cyber Attacks: it is a malicious actor aims to render computer or other device unavailable to its intended users by interrupting the device normal function.

3. Masquerading: It comes out for unauthorized user.

4. Eavesdropping: Intruder communication between sender and receiver

5. Differential: Absorption manner of conducting oneself attitude influence the result to find the intruder for key network transformation.

6. Saturation: it connects for the material and intellectual capability of accredited gathering 


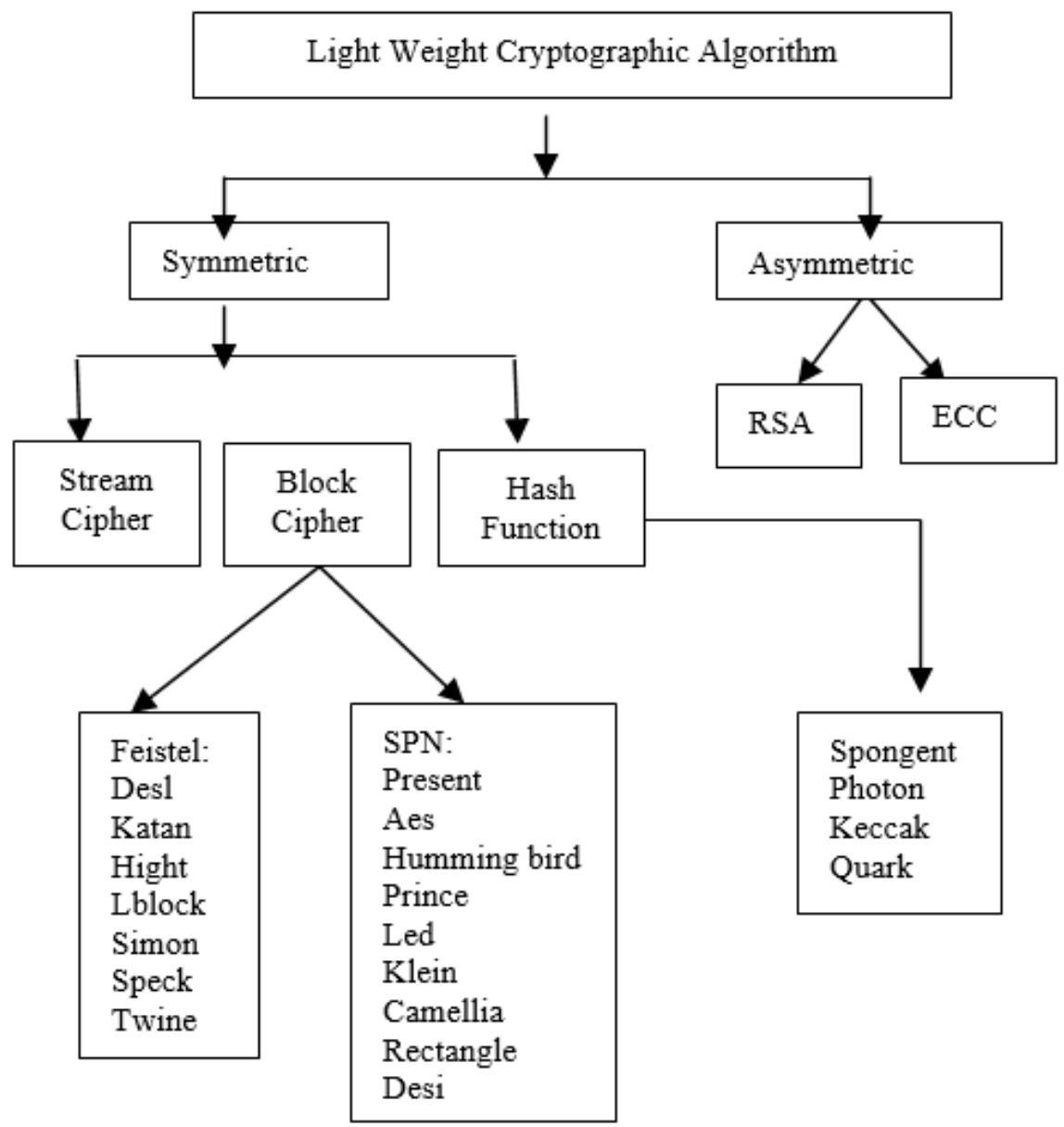

Fig 2. Classification of Lightweight Algorithm

\section{Symmetric Key Cryptography:}

Block Ciphers as the AES have many blocks ciphers with lightweight properties have been invoked. From these PRESENT \& CLEFIA are viewed in detail of implementation and security.

\section{Stream Ciphers:}

A promising new stream ciphers is selected from ECRYPT II eSTREAM Project which taken in between 2004 to 2008. The current eSTREAM portfolio contains 7 algorithms as Trivium, Grain V1 and MICKEY V2 .

\section{Secure Hash Algorithm -3:}

It is a routine purpose hash objective which delights the lightweight equity. These formulas will communicate at various levels .Lightweight hash function based on light weight block cipher can be constructed more security.

\section{E.. Asymmetric Key Cryptography:}

As from the public key primitives is bigger that of symmetric key .lightweight public keys are used for key management process in IoT networks. At this point of view ,No other promising primitives of lightweight and security properties when compared with conventional primitives of RSA \& ECC .

\section{RESEARCH PLAN}

The main observation of this Morse code is to preserve the data .As the initiator and receiver will make communicate information without an unauthorized $3^{\text {rd }}$ party perceive or operate on it. To achieve better improved outcome with greater security and negligible utilization of equipment another procedure called "Lightweight Cryptography(LWC)" is used. Which are designed for the constrains devices. Platforms like WSN, FPGA, RFID etc uses these lightweight cryptographic algorithms.

The requirement consideration of selecting lightweight algorithm with smaller key length ,modest block size , simpler and shorter code measure, lesser clock cycles are counted for estimating and analyzing the efficiency of algorithms . 
Blocks transformation ,Symmetric cipher or fixed sized values are 3 kinds uses of lightweight cryptographic calculation .lightweight cipher uses a keyed pseudo-random permutation for more complex architecture which is used for building blocks. Feistel cipher and substitution -permutation networks are two fundamentals design standards of block ciphers .Generally ,block transformation will not make use of substitution ( S-Box) in the frames and or adjusted in the possible things of small Substitution( S-Box) (PRSENT) . Some block transformations are non linear layer which optimizes the similarity non linear volume called as tiny piece cutting into portions substitution ( S-box), this process executed for other basic functions like AND, OR \& XOR .

Symmetric cipher produces a item that unlocks with XORed and it will considers week and strong possibilities which are called as contemporary or non-contemporary . The symmetric cipher takes the input bit as cramped process of its alternate status from Feedback switch record. it is about 2 kinds of Non Linear FSR, the switch registers that as the absorption chunk as a narrow operation result of the past status of linear feedback shift register . it is defining about different Linear FSR like Galosis, Non parcel galois and Fibonacci . likewise the Non-linear feedback shift register which intakes chunk is an result of the non linear function of its previous state. As the smart card applications and RFID. The NFSR has broad preservation with linear feedback shift register across analyzing the information attacks with algorithms generated using NFSR are sponge .

\section{ANALYSIS OF IMPLMENTABLE}

For the following inconsequential discovers to distinguish with related failing in calculating for other stages. The authors presented a thorough and detailed analysis of various existing lightweight cryptographic algorithms and evaluated the advances in the fields of asymmetric, symmetric algorithms and hash functions.

\section{A. Symmetric Algorithms}

1. AES: A different position with fixed length, that endure put into action on Advanced virtual Risc (AVR ) and Graphics Processing Unit (GPU), etc., proves to be better when compared with already achieved hardware results.

2. PRESENT: this tells about the information of using 8-Bytes and Essential of either 10 bytes or 16 Bytes in making the cryptographic result . This total process is calibrated into three sectors . Starting sector of the information moves at exclusive or function with essential from the modulation of the past 8-byte of the outputs are considered for the function .

3. DES: It is Data Encryption Standard is a block cipher algorithm that takes plain text in blocks of 64 bits and converts them to cipher text using keys of 48 bits .It is resulted on recap arrangement view .Its exhibition was diverge through AES, DESXL, DES and DESX, and is discovered that DESL is better for RFID classify. It gives smallest entryway equality generally.

4. PHOTON : It is about the contrasted with mapping function of KECCAK-200, KECCAK-400, and found to give better outcomes. This process shows two-pass global illumination by increasing adequate adaptability in zone yet better basic at fully length handle.

5. HIGHT : square figure was contradict in the middle and Field programme gate array data processing engineering on design and verification, Cyclone-II Quartus and FPGA scalar. Contrasting both, proportion deals to need for a low force and less assets. HIGHT utilizes straightforward rationale and number juggling activities like XOR, bitwise pivot and expansion. It utilizes 128-piece key on 64-piece square length input. The pipeline configuration shows improvement as far as throughput. It takes $60 \%$ less vitality and multiple times higher throughput. HIGHT executes in four stages:
a. Key plan
b. Initial change
c. 32 iterative round activities
d. Final change

6. SPONGENT: which was executed on for optimized applications that requires the absolute lowest cost in the usage of SPONGENT delivered the fully remarkable during bulk .

7. Lblock is based on Feistel structure and it consists of 32 rounds. The Feistel function of L Block consists of XOR with the round sub keys, substitution and permutation. The half the no. of bits of the input block are revolve in eight bits in each semicircular area. The shape keeps output not in a exceptional tools ability in maintain software effectiveness . The Choice of morse code is a basic and un possible numeric aggression .

8. Hummingbird : it process about the accuracy and speed. It is been executed on the integrated ATAM893-D of Atmel MARC4. The calculation is contrasted with PRESENT, location goes outcomes shows that it gives preferable exhibition over PRESENT on track stage, yet at the same time it can't be shielded from side channel assault, which should be worked in future. 
9. TEA: Limited encipher result is a block cipher mode calculation dependent on ARX level. It is an Assistant determination calculation acquire 2 create: Statistical models Computation for different Computer Performance and two level force utilization is various computer performance. This is contrasted with Round Robin, SEA, EA \& GEA. TEA is the subsequent best to acquire vitality productive server.

10. LEA : A Scrutinize of low inconsequential code Algorithm in this a square figure was executed on Altera Cyclone-III arrangement, Verilog and Xilinx Virtex 5 arrangement. It was contrasted with AES, Hummingbird, PRESENT, Katan, DESL and LED

The creators have expected 2 plan in a equipment usage: speed-select and region structure. The correlation of the two executions infers that speed-select rendition is compelling however not the best in throughput, yet territory is the best in throughput.

11. Robin : is a 128-bits is a deterministic algorithm operating on fixed length group of bits which is feasible choice for software applications. It uses the substitution diffusion techniques.

12. TWINE : calculation has variations 64/80/128-piece square size and was executed on both programming and equipment. It was contrasted with HIGHT, AES, Piccolo and PRESENT. It is intended to fit very little equipment. TWINE figures out how to give viable outcomes on programming. In spite of the fact that it is strong to numerous assaults, Impossible Saturation Morse code and differential cryptography abuse the channel timetable in A lightweight versatile block cipher

13. SPECK : calculation has variations 64/80/128-piece square size and was executed on both programming and equipment. It was contrasted with HIGHT, AES, Piccolo and PRESENT. It is intended to fit very little equipment. TWINE figures out how to give viable outcomes on programming. In spite of the fact that it is strong to numerous assaults, Impossible Saturation cryptography and differential Morse code abuse the channel timetable in non linear layer

14. Simon: square figure was actualized on ASIC application utilizing AVRATmega 128, FPGA Xilinx Spartan 3, Samsung Exynos 5 double, IntelXeon E5640, and MSP430 microcontrollers. While contrasting and SPECK, TWINE, PRESENT, AES and PRINCE, it is found out that spot and Simon are perfect for heterogeneous systems. They are simpler in execution than AES and are likewise exceptionally proficient.

15. Piccolo: is actualized utilizing symmetric structure of the 4 for 16bit leaves . To build dispersion consist of data processing part and key scheduling part in utilizing a byte change among adjusts. Multi level substitution ( S-box ) sheet isolated by a dispersion lattice is utilized in Feistel work. The conceivable assault on Piccolo is a Meet in the Middle assault.

16. PRINCE : has SPN structure and it is used for low resource hardware performance. It has 12 rounds and uses 64bit key called PRINCE core. PRINCE applies distinctive stuff called $\alpha$-reflection.

17. CLEFIA: is block cipher which uses Feistel structure. It uses 128bit, 192 bit, and 256 bit Key on plaintext blocks of size 4096 bits, 8192 bits, 16384 bits, and 24576 bits. For encryption, it uses two diffusion matrixes and two Feistel functions and for decryption it uses round keys and key selection process.

18. RECTANGLE : has SPN structure and it is uses 120 bit key on 64 bit blocks and need 25 rounds for decipherment or decipherment. RECTANGLE can be implemented faster. It uses bit slice technique and is hardware friendly and gives high throughput

19. CAMELLIA : has Fiestel network structure. This functions on 16 bytes blocks and uses 16 byte un locker . It needs 18 rounds for encryption or decryption. It resistant to many passwords and passphrases brute on direction and have high protection positions proportionate for advanced encryption standard.

20. RC5 : was introduced before the lightweight cipher term was introduced and it was extensively used for WSN. It uses Feistel network Structure and works based on data dependent rotations. It operates on two different 32 bit block words, uses 16 byte key and needs 20 rounds.

21. LED : It uses PRESENT cipher Substitution ( s-box) and provides good performance on software aspects. LED operates on 64 bits fixed length string bits of dissimilar key sizes of 128 bits, 96 bits, 80 bits, and 64 bits.

22. PRINT : is a SPN based fixed length group of bits that functions on 6 blocks and 10Bytes of key. It consists of 48 rounds. When the PRINT cipher is of 96 bi block size it uses a 20 byte key and exists of 96 rounds.

23. KATAN : is a lightweight cryptographic block cipher which uses three block ciphers. It uses a feedback, shift and key expansion mechanism which goes through 254 rounds. It uses a non linear update function. 
24. BORON: is a block cipher which uses substitution permutation network. It operates on block length of 8 bytes with 80 or 16 bytes essential length and 25 rounds. BORON uses substitution permutation and 25 different keys are generated from the key.

25. KLEIN : is block cipher. Which uses typical Substitution-Permutation Network (SPN). The possible attack is based on the pre computation

\section{B.Asymmetric Algorithms:}

1. RSA: It was imagined by Ron Rivest, Adi Shamir and Leonard Adleman in 1978. RSA takes a shot at creating open and confidential essential combination by choosing 2 huge best part of existence count . Discover their modulus and picking aimlessly specifies the transformation of plaintext into cipher text and accordingly ascertaining the unscrambling important . the important notation is distributed straightforwardly while cryptographic key is formed protected. An increasingly safe RSA encoder is expected in that is utilized for scramble \& decode records in keeping up protection of client.

2. ECC:. Equal Character is actualized on AT94 k family FPGA and 8-piece integrated. ECC is contrasted with various comparative thin balanced secure communications results that are HIGHT, CLEFIA, DESXL, PRESENT, and so on.

3. Diffie-Hellman - It is about the procedure in getting because it uses a very small secret key. The short key length subjects it to additional ambush and the whole operation is been endangered to eavesdropping attack.

4. Digital Signature Algorithm (DSA) - Though approach is made available covering additional advantages than other generation protocol, it is found to be complex because of the short life span of the digital signatures. Sharing the digital signatures is found to be complicated.

TABLE 2: Performance of Light Weight Algorithms

\begin{tabular}{|c|c|c|c|c|c|c|c|l|}
\hline Algorithm & $\begin{array}{c}\text { Key } \\
\text { Length }\end{array}$ & $\begin{array}{c}\text { Block } \\
\text { Size }\end{array}$ & $\begin{array}{c}\text { No of } \\
\text { Rounds }\end{array}$ & Structure & $\begin{array}{c}\text { Area } \\
\text { Req }\end{array}$ & $\begin{array}{c}\text { Power } \\
\text { Req( } \boldsymbol{\mu W})\end{array}$ & $\begin{array}{l}\text { Through } \\
\text { Put }\end{array}$ & Characteristics \\
\hline AES & 128 & 128 & 10 & SPN & 2400 & 2.48 & 56.64 & Fast, huge key size \\
\hline PRESENT & 80.128 & 64,128 & 32 & SPN & 1030 & 1.54 & 12.4 & $\begin{array}{l}\text { Ultra LWC, Energy } \\
\text { Efficient }\end{array}$ \\
\hline DESL & 184 & 64 & 144 & Feistel & 1848 & 1.6 & 44.4 & $\begin{array}{l}\text { Weakness exploited of } \\
\text { DES ,RFID tags processed } \\
\text { for minimum GE counts }\end{array}$ \\
\hline PHOTON & - & 256 & - & - & 1067 & 14 & 2.82 & $\begin{array}{l}\text { Round function on } \\
\text { lgorithms area wise }\end{array}$ \\
\hline HIGHT & 128 & 64 & 32 & Feistel & 3048 & 5.48 & 188.2 & $\begin{array}{l}\text { ULW,Security,RFID } \\
\text { tagging,greater } \\
\text { throughput/area }\end{array}$ \\
\hline $\begin{array}{c}\text { SPONGEN } \\
\text { T }\end{array}$ & - & 88 & - & LFSR & 738 & 1.57 & 0.81 & $\begin{array}{l}\text { Resistant against } \\
\text { differential attacks }\end{array}$ \\
\hline KECCAK & - & 160 & - & - & 5060 & 11.5 & 14.4 & - \\
\hline QUARK & - & 128 & - & - & 1379 & 2.44 & 1.47 & Min memory requirement \\
\hline LBLOCK & 80 & 64 & 255 & Feistel & 1320 & - & 200 & $\begin{array}{l}\text { Secure-cryptanalysis, } \\
\text { linear cryptanalysis }\end{array}$ \\
\hline $\begin{array}{c}\text { HUMMIN } \\
\text { GBIRD }\end{array}$ & 256 & 16 & 4 & SPN & 2156 & - & 80 & $\begin{array}{l}\text { RFID tags, low power } \\
\text { consumption, high speed }\end{array}$ \\
\hline TEA & 128 & 64 & 32 & Feistel & 2156 & - & 80 & $\begin{array}{l}\text { Security Enhanced, No.of } \\
\text { iterations }\end{array}$ \\
\hline $\begin{array}{c}\text { TWINE } \\
80\end{array}$ & 80 & 64 & 12 & Feistel & 1866 & 1.3 & 178 & $\begin{array}{l}\text { Efficient S/w } \\
\text { performance,ULW ,Enou } \\
\text { gh Speed }\end{array}$ \\
\hline SPECK & 128 & 64 & 34 & Feistel & 1396 & 1.4 & 12.1 & $\begin{array}{l}\text { Key } \\
\text { Recovery ,Boomerang } \\
\text { attack }\end{array}$ \\
\hline PICCOLO- \\
\hline 128 & 64 & 25 & Feistel & 1500 & 0.7 & 0.677 & $\begin{array}{l}\text { Significant improve in } \\
\text { terms of area \& speed }\end{array}$ \\
\hline
\end{tabular}




\begin{tabular}{|c|c|c|c|c|c|c|c|l|}
\hline SIMON & 80 & 32 & 254 & LFSR & 1317 & 1.32 & 22.9 & $\begin{array}{l}\text { Many key sizes, performs } \\
\text { h/w ,performance, easy to } \\
\text { implementation }\end{array}$ \\
\hline CLEFIA & 128 & 128 & 18 & Fiestel & 2488 & 2.48 & 29 & $\begin{array}{l}\text { Fast Encryption and } \\
\text { Decryption, Energy }\end{array}$ \\
\hline $\begin{array}{c}\text { RECTANGL } \\
\text { E }\end{array}$ & 120 & 64 & 25 & SPN & 3048 & 5.48 & 188.2 & $\begin{array}{l}\text { Fast implement by bit } \\
\text { slice techq }\end{array}$ \\
\hline CAMELLIA & 128 & 128 & 18 & SPN & 6511 & 1.54 & 290.1 & $\begin{array}{l}\text { Electronic transformation } \\
\text { of many passwords and } \\
\text { passphrases }\end{array}$ \\
\hline PRINT & 128 & 64 & 13 & SPN & 5.1 & 16.4 & 1.15 & Effective key length \\
\hline
\end{tabular}

1. Memory Requirement:

\section{RESULTS}

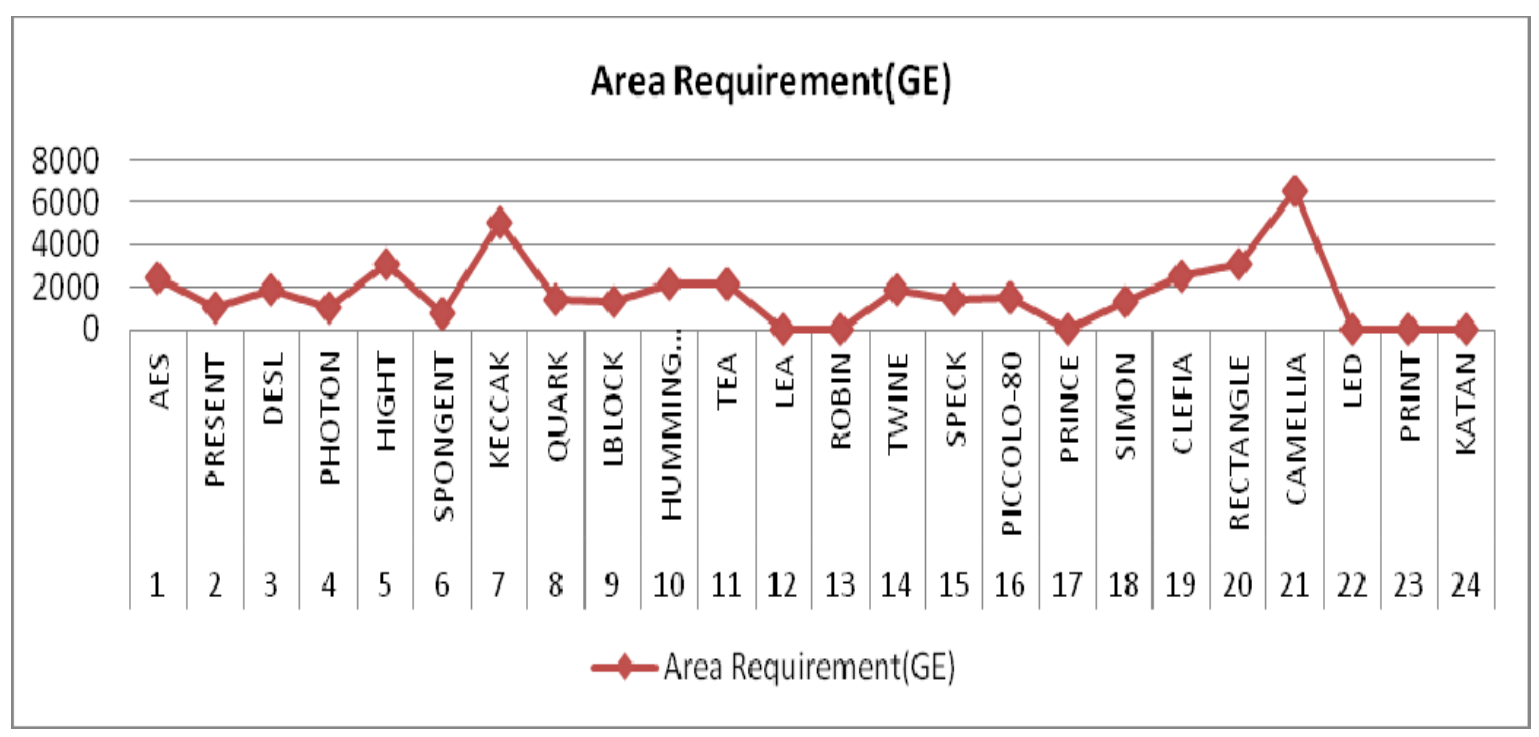

Fig: 3: Represents the Area Requirement for Memory

\section{Energy Consumption:}

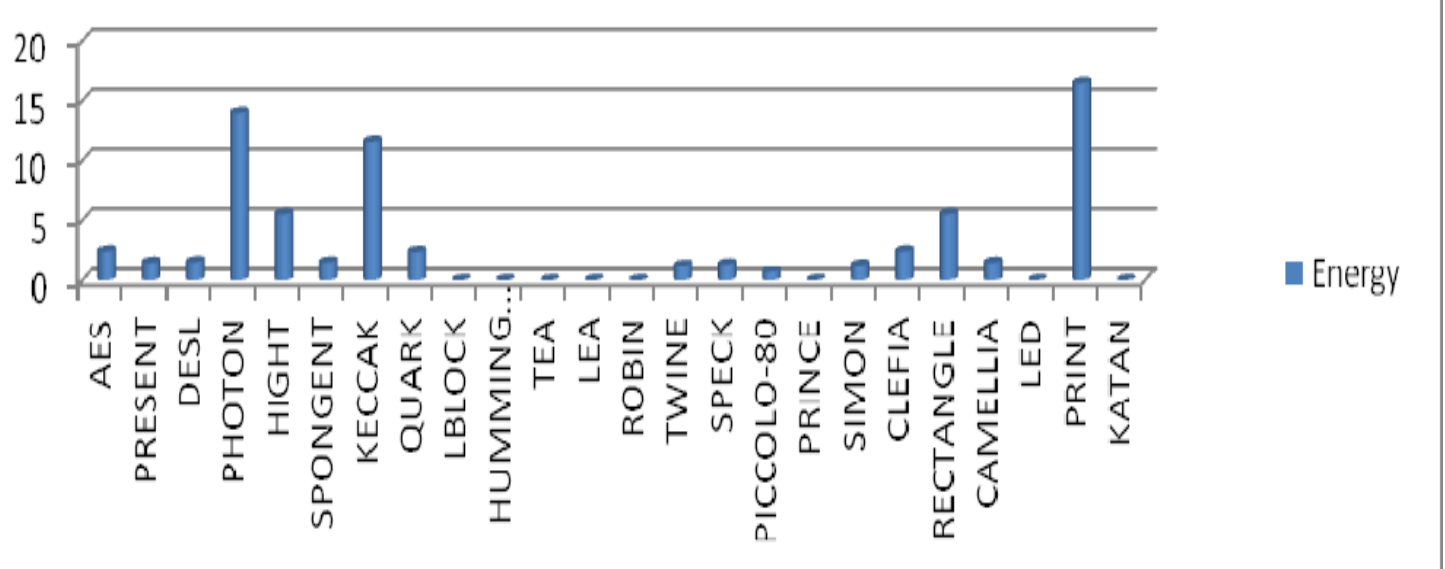

Fig 4. Represents Energy Consumption 


\section{Execution Speed:}

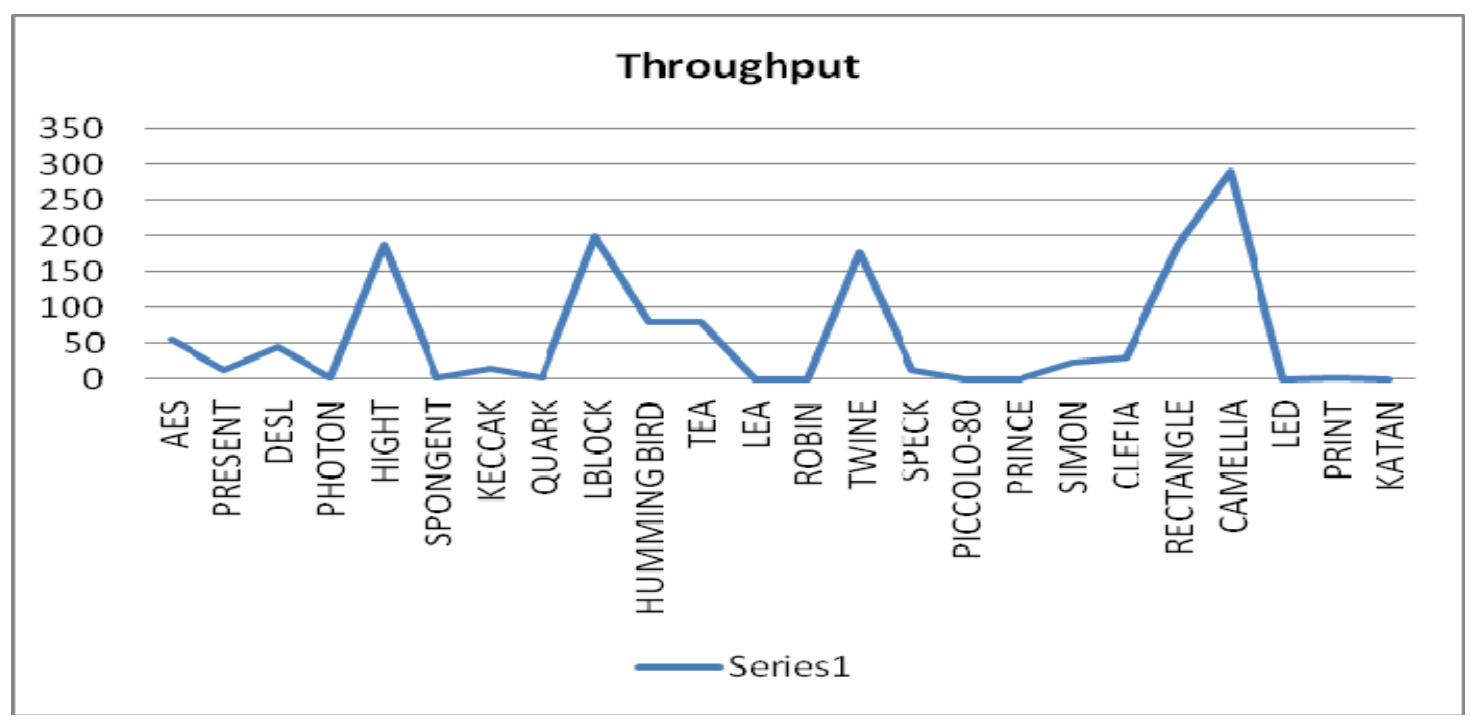

Fig 5 : Represents the Execution Speed

From the parameters displayed about combining the hash algorithm with symmetric algorithm might be an effective way to achieve overall effectiveness

\section{CONCLUSION}

From this paper, we are associating foremost of inconsequential encryption with set of rules to be followed in calculation are investigate in condition near to rules and selections requirement and preservation. Analysed different asymmetric cryptographic algorithm and symmetric cryptographic algorithm for IoT .In this paper we were using LW block cipher uniform design taken into the process for crypto graphy and every level having its assets. As we move with well -designed linear operations, higher security can be provide the price will be authorized based on the structure.

\section{ACKNOWLEDGEMENT}

This is work is supported by Sangam Laxmibhai Vidyapeet education since from 1952, a registered voluntary social action group working for empowering of Women and girls through education.

\section{REFERENCES}

[1] S. M. Metev and V. P. Veiko, Laser Assisted Microtechnology, 2nd ed., R. M. Osgood, Jr., Ed. Berlin, Germany: Springer-Verlag, 1998.

[2] J. Borghoff, et al., "PRINCE - A Low-Latency Block Cipher for Pervasive Computing Applications," in Advances in Cryptology ASIACRYPT, in Springer Berlin Heidelber, vol. 7658, pp. 208-225, July 2013

[3] Shibutani, Takanori Isobe, Harunaga Hiwatari, Atsushi Mitsuda, Toru Akishita, and Taizo ShiraiB. Preneel and T. Takagi, "Piccolo: An Ultra- Lightweight Blockcipher “, in International Association for Cryptologic Research, LNCS 6917, pp. 342-357, Dec 2012.

[4] Xiong Li, Zhou Xuan, Liu Wen "Research on the Architecture of Trusted Security System Based on the Internet of Things" 2011 Fourth International Conference on Intelligent Computation Technology and Automation

[5] Conner, Margery (May 27 2010). Sensors empower the "Internet of Things" pp. 32-38. ISSN 0012-7515

[6] Mayuri A. Bhabad, Sudhir T. Bagade "Internet of Things: Architecture, Security Issues and Countermeasures", International Journal of Computer Applications (0975 - 8887) Volume 125 - No.14, September 2015

[7] Bos, J.W., Osvik, D.A., Stefan, D.: Fast implementations of AES on various platforms. IACR (2009)

[8] Rolfes,C., Poschmann, A., Leander, G., Paar,C.: Ultra-Lightweight Implementations for Smart Devices-Security for 1000 Gate Equivalents. Springer, Germany (2008)

[9] Fan, X., Hu, H., Gong, G., Smith, E.M., Engels, D.: Lightweight Implementation of Hummingbird Cryptographic Algorithm on 4-Bit Microcontrollers. IEEE (2009)

[10] Ghafari, V.A., Hu, H., Chen, Y.: Fruit-v2: ultra-lightweight stream cipher with shorter internal state. Int. Assoc. Cryptol. Res (IACR) (2016)

[11] Jungk, B., Lima, L.R., Hiller, M.: A Systematic Study of Lightweight Hash Functions on FPGAs. IEEE (2014)

[12] Aumasson, J.-P., Henzen, L., Meierm, W., Naya-Plasencia, M.: Quark: a lightweight hash. CHES (2010)

[13] Bertoni, G., Daemon, J., Peeters, M., Van Assche, G.: Sponge Functions, ECRYPT hash workshop (2007)

[14] Panasenko, S., Smagin, S.: Lightweight cryptography: underlying principles and approaches. Int. J. Comput. Theory Eng. 3 (2011) A Survey of Lightweight Cryptographic Algorithms ... 293

[15] McKay, K.A., Bassham, L., Turan, M.S., Mouha, N.: Report on lightweight Cryptogaphy. National Institute of Standards and Technology Internal Report 8114 (2017)

[16] Diehl,W., Farahmand, F., Yalla, P., Kaps, J.-P., Gaj, K.:Comparison of Hardware and Software Implementations of Selected Lightweight Block Ciphers. IEEE (2017)

[17] Leander, G., Paar, C., Poschmann, A., Schramm, K.:Newlightweight DES variants. Int. Assoc. Cryptol. Res. (2007) 
[18] Mohd1, B.J., Hayajneh, T., Khalaf, Z.A., Yousef, K.M.A.: Modeling and optimization of the lightweight HIGHT block cipher design with FPGA implementation. Security and Communication Networks. Wiley (2016)

[19] Kataoka, H., Sawada, A., Duolikun, D., Enokido, T.: Energy-aware server selection algorithms in a scalable cluster. In: International Conference on Advanced Information Networking and Applications. IEEE (2016)

[20] Lee, D.,Kim,D.-C.,Kwon, D.,Kim,H.: Efficient Hardware Implementation of the Lightweight Block Encryption Algorithm LEA. Sensors (2014)

[21] Beaulieu, R., Shors, D., Smith, J., Treatment-Clark, S., Weeks, B., Wingers, L.: Simon and Speck: Block Ciphers for the Internet of Things. NIST Lightweight Cryptography (2015)

[22] Duka, A.V., Genge, B.: Implementation of SIMON and SPECK Lightweight Block Ciphers on Programmable Logic Controllers. IEEE (2017)

[23] Suzaki, T., Minematsu, K., Morioka, S., Kobayashi, E.: TWINE: A Lightweight Block Cipher for Multiple Platforms. Springer (2012)

[24] Sicari S, Cappiello C, Pellegrini F, Miorandi D,Coen-Prisini A "A security-and quality-aware system architecture for internet of things", Information Systems Frontiers, Vol.6, no.3, March 2014.

[25] J.-P. Aumasson, L. Henzen, W. Meier, and M. Naya-Plasencia, "Quark: A Lightweight Hash.” in CHES 2010, no. 6225 in LNCS, pp. $1-15$, Springer-Verlag, 2010.

[26] A. Bogdanov, L. R. Knudsen, G. Leander, C. Paar, A. Poschmann, M. J. B. Robshaw, Y. Seurin, and C. Vikkelsoe, "PRESENT: An Ultra-Lightweight Block Cipher." in CHES 2007, no. 4727 in LNCS, pp. 450-466, Springer-Verlag, 2007.

[27] S Pradeep, Dr Yogesh kumar Sharma 2019. Storing Live sensor data to the platforms of IOT using Arduino and Associated Microchips, Springer ,ISSN: 2194-5337, pp:1-15.

\section{AUTHOR PROFILE}

Dr S Pradeep, Presently working as Associate Professor in the Department of Computer Science and Engineering, Bhoj Reddy Engineering College for Women, Hyderabad, Telangana ,India. He has completed $\mathrm{PhD}$ from Shri Jagdishprasad Jhabarmal Tibrewala University in the year 2019, Jhunjhunu, Rajasthan and Completed Master's and Bachelor's Degree from JPNCE, Mahabubnagar, Telangana in the year 2012 and 2010. His area of research towards IOT, Wireless sensor Networks, Cloud, Security and Block chain . He is the member of professional bodies of IET, IEEE, ACM \& IAENG. 